\title{
ON THE THEOREM OF BOREL FOR QUASIANALYTIC CLASSES
}

\author{
JOSÉ BONET and REINHOLD MEISE
}

Dedicated to the memory of our friend Klaus D. Bierstedt

\begin{abstract}
We investigate the surjectivity of the Borel map in the quasianalytic setting for classes of ultradifferentiable functions defined in terms of the growth of the Fourier-Laplace transform. We deal with both the Roumieu $\mathscr{E}_{\{\omega\}}$ and the Beurling $\mathscr{E}_{(\omega)}$ classes for a weight function $\omega$. In particular, we show that a classical result of Carleman for the quasianalytic classes $\mathscr{E}_{\left\{M_{p}\right\}}$ also holds for the classes defined using weights. We also characterize when the space of quasianalytic germs at the origin coincides with the space of real analytic germs at the origin.
\end{abstract}

1. Introduction. Classes of ultradifferentiable functions on $\mathrm{R}^{n}$ are usually defined by imposing conditions on the derivatives of the functions. See, e.g., Komatsu [8] for the definition of the classes $\mathscr{E}_{\left\{M_{p}\right\}}\left(\mathrm{R}^{n}\right)$ and $\mathscr{E}_{\left(M_{p}\right)}\left(\mathrm{R}^{n}\right)$ of Roumieu and Beurling type respectively associated with a sequence of positive numbers $\left(M_{p}\right)_{p}$. Continuing the classical work of Borel, many authors have investigated conditions on the sequence $\left(M_{p}\right)_{p}$ and on a sequence $\left(a_{\alpha}\right)_{\alpha \in \mathrm{N}_{0}^{n}}$ to ensure the existence of a function $f$ in the class associated with $\left(M_{p}\right)_{p}$ such that $f^{(\alpha)}(0)=a_{\alpha}$ for each $\alpha \in \mathrm{N}_{0}^{n}$. See Meise and Taylor [9] and Petzsche [11] and the references therein. A class of ultradifferentiable functions is called quasianalytic if the Borel map $B(f):=\left(f^{(\alpha)}(0)\right)_{\alpha \in \mathrm{N}_{0}^{n}}$ is injective. A classical theorem of Carleman shows the non-surjectivity of the Borel map in the quasianalytic non real analytic case. A simplified, comprehensive proof of Carleman's result is presented by Thilliez [14] Theorem 3.

In this paper we investigate the Borel map for quasianalytic classes $\mathscr{E}_{\{\omega\}}$ and $\mathscr{E}_{(\omega)}$ for a weight function $\omega$ defined in terms of the growth of the FourierLaplace transform, like in the work of Beurling and Björck. We work in the context of ultradifferentiable functions as defined by Braun, Meise and Taylor [4]. A precise comparison of the two ways to define ultradifferentiable classes was given by Bonet, Meise and Melikhov in [3]. Borel's theorem for non quasianalytic classes of Beurling type $\mathscr{E}_{(\omega)}$ was investigated by Meise and Taylor [9] and for non quasianalytic classes of Roumieu type by Bonet, Meise and

Received 19 May 2011. 
Taylor in [1]. This research was continued in [2], where the investigation of the range of the Borel map, when it is not surjective, required quasianalytic classes. In our main result Theorem 14 we characterize those weights $\omega$ such that the Borel map is surjective on germs of quasianalytic functions of Roumieu type $\mathscr{E}_{\{\omega\}, 0}(n)$ onto the natural sequence space $\Lambda_{\{\omega\}}(n)$. This is a version of Carleman's theorem in the context of classes defined using weights, that permits us to conclude in Corollary 16 that the Borel map is never surjective on the space $\mathscr{E}_{\{\omega\}}(\Omega)$. This result shows that the statement of Chung and Kim [5] Theorem 3.2 is not correct. As a main step in the proof of Theorem 14, we characterize the quasianalytic weight functions $\omega$ such that the space of real analytic germs $\mathscr{O}_{0}(n)$ is strictly contained in the space of quasianalytic germs $\mathscr{E}_{\{\omega\}, 0}(n)$ at the origin. Our proofs are completely different from those of Thilliez [14]. We use the Fourier-Laplace transform to work on weighted spaces of entire functions and apply functional analytic methods together with a theorem of Hörmander (Theorem 12 about the existence of entire functions with prescribed growth). The corresponding results for germs of quasianalytic functions of Beurling type are presented in Proposition 18 and Theorem 19.

We refer the reader to Meise, Vogt [10] for undefined terminology on functional analysis, to Hörmander [6] for complex analysis and to Braun, Meise and Taylor [4] and Komatsu [8] for ultradifferentiable functions.

2. Definition. A function $\omega:[0, \infty[\rightarrow[0, \infty$ [ is called a weight function if it is continuous, increasing, and satisfies $\omega(0)=0$ and the following conditions

$(\alpha)$ There exists $K \geq 1$ such that $\omega(2 t) \leq K \omega(t)+K, t \geq 0$.

$(\beta) \omega(t)=O(t)$ as $t$ tends to infinity

$(\gamma) \log (t)=o(\omega(t))$ as $t$ tends to infinity

( $\delta) \phi: t \mapsto \omega\left(e^{t}\right)$ is convex on $[0, \infty[$.

If the weight function $\omega$ satisfies

$$
\int_{1}^{\infty} \frac{\omega(t)}{t^{2}} d t=\infty
$$

then it will be called a quasianalytic weight function. Otherwise it is called non-quasianalytic.

The function $\tilde{\omega}: \mathrm{C}^{n} \rightarrow[0, \infty[, \tilde{\omega}(z):=\omega(|z|)$ will also be denoted by $\omega$.

The Young conjugate of $\phi=\phi_{\omega}$, defined in $(\delta)$, is given by

$$
\phi^{*}(x):=\sup \{x y-\phi(y): y>0\}, \quad x \geq 0 .
$$

3. EXAMPLE. The following functions are easily seen to be quasianalytic weight functions: 
(1) $\sigma(t):=t$

(2) $\omega(t):=t(\log (e+t))^{-\alpha}, 0<\alpha<1$

(3) $\omega(t):=t(\log (e+\log (e+t)))^{-\beta}, \beta>0$.

4. Definition. For an open set $\Omega$ in $\mathrm{R}^{n}$ and for a weight function $\omega$ we define:

(a) $\mathscr{E}_{\{\omega\}}(\Omega):=\left\{f \in C^{\infty}(\Omega)\right.$ : For each $K \subset \Omega$ compact there exists $m \in \mathrm{N}$ :

$$
\left.\|f\|_{K, m}:=\sup _{x \in K} \sup _{\alpha \in \mathrm{N}_{0}^{n}}\left|f^{(\alpha)}(x)\right| \exp \left(-\frac{1}{m} \phi^{*}(m|\alpha|)\right)<\infty\right\}
$$

and we endow it with the usual projective topology over $K$ of the inductive topology over $m$. The elements of $\mathscr{E}_{\{\omega\}}(\Omega)$ will be called $\{\omega\}$-ultradifferentiable functions of Roumieu type on $\Omega$.

(b) $\mathscr{E}_{(\omega)}(\Omega):=\left\{f \in C^{\infty}(\Omega)\right.$ : For each $K \subset \Omega$ compact and each $m \in \mathrm{N}$ :

$$
\left.p_{K, m}(f):=\sup _{x \in K} \sup _{\alpha \in \mathrm{N}_{0}^{n}}\left|f^{(\alpha)}(x)\right| \exp \left(-m \phi^{*}\left(\frac{|\alpha|}{m}\right)\right)<\infty\right\}
$$

and we endow it with its natural Fréchet space topology. The elements of $\mathscr{E}_{(\omega)}(\Omega)$ are called $(\omega)$-ultradifferentiable functions of Beurling type on $\Omega$.

A weight $\omega$ is quasianalytic if and only if a function $f$ in any of the classes $\mathscr{E}_{\{\omega\}}(\Omega)$ or $\mathscr{E}_{(\omega)}(\Omega)$ is identically 0 as soon as the sequence of all the derivatives at a point in a connected open set $\Omega$ vanishes.

REMARK. Note that for $\sigma(t)=t$ we get for each open set $\Omega$ in $\mathrm{R}^{n}$ that $\mathscr{E}_{\{\sigma\}}(\Omega)$ is just the space of all real-analytic functions on $\Omega$ which usually is denoted by $\mathscr{A}(\Omega)$. Moreover, $\mathscr{E}_{(\sigma)}(\Omega)=H\left(C^{n}\right)$ for each open set $\Omega$, via the restriction of the entire functions to $\Omega$.

5. Definition. For a weight function $\omega$ we define:

(a) The spaces of germs of the $\omega$-ultradifferentiable functions at $0 \in \mathrm{R}^{n}$ by

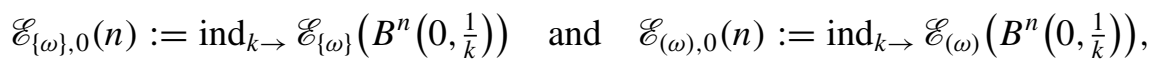
where $B(a, r):=\left\{x \in \mathrm{R}^{n}:|x-a|<r\right\}$.

(b) The sequence spaces

$$
\begin{aligned}
\Lambda_{\{\omega\}}(n):=\left\{\left(a_{\alpha}\right)_{\alpha \in \mathrm{N}_{0}^{n}} \in \mathrm{C}^{\mathrm{N}_{0}^{n}}: \text { There exists } m \in \mathrm{N}:\right. \\
\left.\qquad a \|_{m}:=\sup _{\alpha \in \mathrm{N}_{0}}\left|a_{\alpha}\right| \exp \left(-\frac{1}{m} \phi^{*}(m|\alpha|)\right)<\infty\right\}
\end{aligned}
$$

and

$$
\begin{aligned}
& \Lambda_{(\omega)}(n):=\left\{\left(a_{\alpha}\right)_{\alpha \in \mathrm{N}_{0}^{n} \in \mathrm{C}^{\mathrm{N}_{0}^{n}}}: \text { For each } m \in \mathrm{N}:\right. \\
& \left.\qquad p_{m}(a):=\sup _{\alpha \in \mathrm{N}_{0}}\left|a_{\alpha}\right| \exp \left(-m \phi^{*}\left(\frac{|\alpha|}{m}\right)\right)<\infty\right\} .
\end{aligned}
$$


Obviously $\Lambda_{\{\omega\}}(n)$ is an (LB)-space, while $\Lambda_{(\omega)}(n)$ is a Fréchet space for each $n \in \mathrm{N}$.

(c) For $\omega(t):=t$, we write $\mathscr{O}_{0}(n)$ instead of $\mathscr{E}_{\{\omega\}, 0}(n)$.

6. Remark. For each weight function $\omega$ and $\phi:=\phi_{\omega}$ the following assertions hold:

(a) For each $m \in \mathbf{N}$ there exist $k \in \mathrm{N}, k>m$, and $C>0$ such that for each $j \in \mathrm{N}: \exp \left(-m \phi^{*}\left(\frac{j}{m}\right)\right) \leq C e^{-j} \exp \left(-k \phi^{*}\left(\frac{j}{k}\right)\right)$.

(b) For each $p \in \mathrm{N}$ there exist $q \in \mathrm{N}, q>p$, and $C>0$ such that for each $j \in \mathrm{N}: \exp \left(\frac{1}{p} \phi^{*}(p j)\right) \leq C e^{-j} \exp \left(\frac{1}{q} \phi^{*}(q j)\right)$.

To prove this, note first that from Condition $2(\alpha)$ we get the existence of $A \in \mathrm{N}$ such that $\omega(t) \leq A \omega(t / e)$ for all large $t>0$. This implies $\phi(x) \leq A \phi(x-1)$ for all large $x>0$ and consequently

$$
\phi^{*}(y) \geq y+A \phi^{*}(y / A) \quad \text { for all large } \quad y>0 .
$$

To prove (a), fix $m \in \mathrm{N}$ and let $k:=A m$. From (1) we get for large $j \in \mathrm{N}$

$$
m \phi^{*}\left(\frac{j}{m}\right) \geq j+k \phi^{*}\left(\frac{j}{k}\right),
$$

which implies (a).

To prove (b) let $p \in \mathrm{N}$ be given and let $q:=A p$. Then we get from (1) that for large $j$ we have

$$
\frac{1}{q} \phi^{*}(q j) \geq j+\frac{1}{p} \phi^{*}(p j)
$$

which implies (b).

7. Lemma. For each weight function $\omega$ and each $n \in \mathrm{N}$ the following holds:

(a) $\Lambda_{(\omega)}(n)$ is a nuclear Fréchet space and its dual can be identified with

$$
\begin{aligned}
& \Lambda_{(\omega)}^{\prime}(n)=\left\{y \in \mathrm{C}_{0}^{n}: \text { There exists } k \in \mathrm{N}:\right. \\
& \left.\qquad y \|_{k}:=\sum_{\alpha \in \mathrm{N}_{0}^{n}}\left|y_{\alpha}\right| \exp \left(k \phi^{*}\left(\frac{|\alpha|}{k}\right)\right)<\infty\right\}
\end{aligned}
$$

under the bilinear form $\langle y, x\rangle:=\sum_{a \in \mathrm{N}_{0}^{n}} x_{\alpha} y_{\alpha}$.

(b) $\Lambda_{\{\omega\}}(n)$ is a (DFN)-space and as in (a) its dual is given by

$$
\begin{aligned}
& \Lambda_{\{\omega\}}^{\prime}(n)=\left\{y \in \mathrm{C}_{0}^{n}: \text { For each } k \in \mathrm{N}:\right. \\
& \left.\qquad p_{k}(y):=\sum_{\alpha \in \mathrm{N}_{0}^{n}}\left|y_{\alpha}\right| \exp \left(\frac{1}{k} \phi^{*}(k|\alpha|)\right)<\infty\right\} .
\end{aligned}
$$

Proof. (a) In the notation of Meise and Vogt [10], §27, we have $\Lambda_{(\omega)}(n)=$ $\lambda^{\infty}(A)$, where the Köthe matrix $A=\left(a_{\alpha, k}\right)_{\alpha \in \mathrm{N}_{0}^{n}, k \in \mathrm{N}}$ is given by $a_{\alpha, k}:=$ 
$\exp \left(-k \phi^{*}\left(\frac{|\alpha|}{k}\right)\right)$. From Remark 6(a) we know that for each $m \in \mathrm{N}$ there exist $k \in \mathrm{N}$ and $C>0$ such that

$$
\sum_{\alpha \in \mathrm{N}_{0}^{n}} \frac{a_{\alpha, m}}{a_{\alpha, k}} \leq C \sum_{\alpha \in \mathrm{N}_{0}^{n}} e^{-|\alpha|}=C \sum_{\alpha_{1}=0}^{\infty} \ldots \sum_{\alpha_{n}=0}^{\infty} e^{-\alpha_{1}} \ldots e^{-\alpha_{n}}=C\left(\sum_{j=0}^{\infty} e^{-j}\right)^{n}<\infty .
$$

By [10], Proposition 28.16, this implies that $\lambda^{\infty}(A)$ is a nuclear Fréchet space. From this and [10], Proposition 27.13, it follows that $\Lambda_{(\omega)}^{\prime}(n)$ has the given form and that the strong topology of $\Lambda_{(\omega)}^{\prime}(n)$ is given by the corresponding inductive limit topology.

(b) If we define the Köthe matrix $B:=\left(b_{\alpha, k}\right)_{\alpha \in \mathrm{N}_{0}^{n}, k \in \mathrm{N}}$ by $b_{\alpha, k}:=\exp \left(\frac{1}{k}\right.$ $\left.\phi^{*}(k|\alpha|)\right)$ then it follows from Remark 6(b) as in part (a) that $\lambda^{1}(B)$ is a nuclear Fréchet space and hence reflexive. From [10], Proposition 27.13, it follows that $\lambda^{1}(B)^{\prime}=\Lambda_{\{\omega\}}(n)$. Since $\lambda^{1}(B)$ is reflexive, this proves (b).

Next we introduce several weighted spaces of entire functions, where we denote the space of all entire functions on $\mathrm{C}^{n}$ by $H\left(\mathrm{C}^{n}\right)$.

8. Definition. Let $\omega$ be a weight function and $\Omega \subset \mathrm{R}^{n}$ a convex open set.

(a) For a compact subset $K$ of $\Omega$ its support functional $h_{K}$ is defined as $h_{K}(x):=\sup _{y \in K}\langle x, y\rangle$.

(b) $A_{\{\omega\}}(\Omega):=\left\{f \in H\left(C^{n}\right)\right.$ : There exists $K \subset \Omega$ compact such that

$$
\left.\|f\|_{K, m}:=\sup _{z \in C^{n}}|f(z)| \exp \left(-h_{K}(\operatorname{Im} z)-\frac{1}{m} \omega(z)\right)<\infty \text { for each } m \in \mathbf{N}\right\} .
$$

(c) $A_{\{\omega\}}(n):=\left\{f \in H\left(\mathrm{C}^{n}\right):\|f\|_{\{0\}, m}<\infty\right.$ for each $\left.m \in \mathrm{N}\right\}$.

(d) $A_{\{\omega\}, 0}(n):=\left\{f \in H\left(C^{n}\right):\right.$ For each $m \in \mathbf{N}$ :

$$
\left.\|f\|_{m}:=\sup _{z \in \mathrm{C}^{n}}|f(z)| \exp \left(-\frac{1}{m}(|\operatorname{Im} z|+\omega(z))\right)<\infty\right\} .
$$

(e) $A_{(\omega)}(\Omega):=\left\{f \in H\left(C^{n}\right)\right.$ : There exist $K \subset \Omega$ compact and $m \in \mathrm{N}$ :

$$
\left.q_{K, m}(f):=\sup _{z \in C^{n}}|f(z)| \exp \left(-h_{K}(\operatorname{Im} z)-m \omega(z)\right)<\infty\right\} .
$$

(f) $A_{(\omega)}(n):=\left\{f \in H\left(\mathrm{C}^{n}\right)\right.$ : There exists $\left.m \in \mathrm{N}: q_{\{0\}, m}(f)<\infty\right\}$.

(g) $A_{(\omega), 0}(n):=\left\{f \in H\left(\mathrm{C}^{n}\right):\right.$ For each $m \in \mathrm{N}$ there exists $k \in \mathrm{N}$ :

$$
\left.\rho_{m, k}(f):=\sup _{z \in \mathrm{C}}|f(z)| \exp \left(-\frac{1}{m}|\operatorname{Im} z|-k \omega(z)\right)<\infty\right\} .
$$

If we endow the spaces in Definition 8 with their natural topology, then the following can be checked by standard arguments: $A_{\{\omega\}}(\Omega)$ is an (LF)-space, 
$A_{\{\omega\}}(n)$ and $A_{\{\omega\}, 0}(n)$ are Fréchet spaces, $A_{(\omega)}(\Omega)$ and $A_{(\omega)}(n)$ are (LB)-spaces, and $A_{(\omega), 0}(n)$ is a projective limit of (LB)-spaces.

Notation. If we use the index $*$ in a statement, this means that the statement is valid if at all places $*$ is replaced by either $(\omega)$ or by $\{\omega\}$. Using this notation we recall the following result from Heinrich and Meise [7], Theorem 3.6 and 3.7, where the Roumieu case was proved already in Rösner [12], Theorem 2.19.

9. THEOREM. For each weight function $\omega$ and each convex open set $\Omega$ in $\mathrm{R}^{n}$ the Fourier-Laplace transform

$$
\mathscr{F}: \mathscr{E}_{*}^{\prime}(\Omega) \rightarrow A_{*}(\Omega), \quad \mathscr{F}(\mu): z \mapsto\left\langle\mu_{x}, \exp (-i\langle x, z\rangle)\right\rangle, \quad z \in \mathrm{C}^{n}
$$

is a linear topological isomorphism.

10. Corollary. For each quasianalytic weight function $\omega$ and each $n \in \mathrm{N}$ the Fourier-Laplace transform

$$
\mathscr{F}: \mathscr{E}_{*, 0}^{\prime}(n) \rightarrow A_{*, 0}(n), \quad \mathscr{F}(\mu): z \mapsto\left\langle\mu_{x}, \exp (-i\langle x, z\rangle)\right\rangle, \quad z \in \mathrm{C}^{n},
$$

is a linear topological isomorphism.

Proof. Since $\omega$ is quasianalytic, the inductive topology of $\mathscr{E}_{*, 0}(n)$ is Hausdorff. Hence $\mathscr{E}_{\{\omega\}, 0}(n)$ is an (LB)-space, while $\mathscr{E}_{(\omega), 0}(n)$ is an (LF)-space.

To prove the assertion in the case $\mathscr{E}_{\{\omega\}, 0}^{\prime}(n)$, note first that by Theorem 9 for each $k \in \mathrm{N}$ the Fourier-Laplace transform

$$
\mathscr{F}_{k}: \mathscr{E}_{\{\omega\}}^{\prime}\left(B\left(0, \frac{1}{k}\right)\right) \rightarrow A_{\{\omega\}}\left(B\left(0, \frac{1}{k}\right)\right)
$$

is a linear topological isomorphism. Hence the induced map

$$
\mathscr{F}: \operatorname{proj}_{\leftarrow k} \mathscr{E}_{\{\omega\}}^{\prime}\left(B\left(0, \frac{1}{k}\right)\right) \rightarrow \operatorname{proj}_{\leftarrow k} A_{\{\omega\}}\left(B\left(0, \frac{1}{k}\right)\right)
$$

is a linear topological isomorphism, too. Obviously, a fundamental system for the continuous semi-norms on $\operatorname{proj}_{\leftarrow k} A_{\{\omega\}}\left(B\left(0, \frac{1}{k}\right)\right)$ is given by $\left(\|\cdot\|_{B(0, \epsilon), m}\right)_{\epsilon>0, m \in N}$. Since the system $\left(\|\cdot\|_{m}\right)_{m \in \mathbb{N}}$ is cofinal in this system, we get that $\operatorname{proj}_{\leftarrow k} A_{\{\omega\}}\left(B\left(0, \frac{1}{k}\right)\right)=A_{\{\omega\}, 0}(n)$.

On the other hand it is easy to check that the inductive spectrum $\left(E_{m}\right.$, $\left.j_{m, p}\right)_{m \in \mathrm{N}}$ of Banach spaces, where

$$
\begin{aligned}
E_{m}:=\left\{f \in C^{\infty}(\overline{B(0,1 / m)}): \sigma_{m}(f)\right. \\
\left.\quad:=\sup _{x \in \overline{B(0,1 / m)}}\left|f^{(\alpha)}(x)\right| \exp \left(-\frac{1}{m} \phi^{*}(m|\alpha|)\right)<\infty\right\}
\end{aligned}
$$


and where $j_{m, p}: E_{m} \rightarrow E_{p}$ denotes the restriction map, is equivalent to the inductive spectrum $\left(\mathscr{E}_{\{\omega\}},\left(B\left(0, \frac{1}{k}\right)\right)\right)_{k \in \mathrm{N}}$. Hence $\mathscr{E}_{\{\omega\}, 0}(n)$ is a (DFS)-space and

$$
\mathscr{E}_{\{\omega\}, 0}^{\prime}(n)=\operatorname{proj}_{\leftarrow m} E_{m}^{\prime}=\operatorname{proj}_{\leftarrow k} \mathscr{E}_{\{\omega\}}^{\prime}\left(B\left(0, \frac{1}{k}\right)\right),
$$

which proves the assertion in the case $\mathscr{E}_{\{\omega\}, 0}^{\prime}(n)$.

For the case $\mathscr{E}_{(\omega), 0}^{\prime}(n)$ the proof can be given similarly, again using Theorem 9 in the steps.

11. Proposition. For each weight function $\omega$ and each $n \in \mathrm{N}$ the map

$$
T: \Lambda_{*}^{\prime}(n) \rightarrow A_{*}(n), T(y):=\sum_{\alpha \in \mathrm{N}_{0}^{n}}(-i)^{|\alpha|} y_{\alpha} z^{\alpha}, z \in \mathrm{C}^{n},
$$

is a linear topological isomorphism.

Proof. To show that $T$ is well-defined on $\Lambda_{(\omega)}^{\prime}(n)$, note that by Lemma 7(a) we have

$$
\begin{aligned}
\Lambda_{(\omega)}^{\prime}(n)=\left\{y \in \mathrm{C}^{\mathrm{N}_{0}^{n}}: \text { There exists } k\right. & \in \mathrm{N}: \\
\|y\|_{k} & \left.:=\sum_{\alpha \in \mathrm{N}_{0}^{n}}\left|y_{\alpha}\right| \exp \left(k \phi^{*}\left(\frac{|\alpha|}{k}\right)\right)<\infty\right\} .
\end{aligned}
$$

Now fix $y \in \Lambda_{(\omega)}^{\prime}(n)$ and choose $m \in \mathrm{N}$ such that $\|y\|_{m}<\infty$. Then we get

$$
\begin{aligned}
q_{\{0\}, m}(T(y)) & =\sup _{z \in C^{n}}\left|\sum_{\alpha \in \mathrm{N}_{0}^{n}}(-i)^{|\alpha|} y_{\alpha} z^{\alpha}\right| \exp (-m \omega(z)) \\
& \leq \sum_{\alpha \in \mathrm{N}_{0}^{n}}\left|y_{\alpha}\right| \exp \left(\sup _{z \in \mathrm{C}^{\mathrm{N}}}(|\alpha| \log |z|-m \omega(z))\right) \\
& \leq \sum_{\alpha \in \mathrm{N}_{0}^{n}}\left|y_{\alpha}\right| \exp \left(m\left(\sup _{\xi \geq 0}\left(\frac{|\alpha|}{m} \xi-\phi(\xi)\right)\right)\right) \\
& \leq \sum_{\alpha \in \mathrm{N}_{0}^{n}}\left|y_{\alpha}\right| \exp \left(m \phi^{*}\left(\frac{|\alpha|}{m}\right)\right)=\|y\|_{m} .
\end{aligned}
$$

Hence $T(y)$ is in $A_{(\omega)}(n)$. Moreover, this estimate shows that the map $T$ is continuous. Since $T$ is obviously injective, it remains to show that $T$ is surjective. To prove this, let $f \in A_{(\omega)}(n)$ be given. Then there exists $m \in \mathbf{N}$ such that $q_{\{0\}, m}(f)<\infty$. Next we consider the Taylor expansion of $f$,

$$
f(z)=\sum_{\alpha \in \mathrm{N}_{0}^{n}} f_{\alpha} z^{\alpha},
$$

where

$$
f_{\alpha}:=\left(\frac{1}{2 \pi}\right)^{n} \int \ldots \int_{\left|\zeta_{j}\right|=r} \frac{f(\zeta)}{\zeta_{1}^{\alpha_{1}+1} \ldots \zeta_{n}^{\alpha_{n}+1}} d \zeta_{1} \ldots d \zeta_{n}
$$


for any number $r>0$. Since $\omega$ satisfies Condition 2( $\alpha)$, we can choose $p \in \mathbf{N}$ and $c>0$ such that $\omega(\sqrt{n} t) \leq p \omega(t)+c$. Using this we can estimate $f_{\alpha}$ for $r>1$ as follows:

$$
\begin{aligned}
\left|f_{\alpha}\right| & \leq \frac{1}{r^{|\alpha|}} \exp \left(m \omega\left(r e^{i t}, \ldots, r e^{i t}\right)\right) q_{\{0\}, m}(f) \\
& \leq q_{\{0\}, m}(f) \exp (-|\alpha| \log r+m \omega(\sqrt{n} r)) \\
& \leq q_{\{0\}, m}(f) e^{m c} \exp (-|\alpha| \log r+m p \omega(r)) .
\end{aligned}
$$

Since this estimate holds for each $r>1$, we can take the infimum over $r>1$ and get

$$
\left|f_{\alpha}\right| \leq e^{m c} q_{\{0\}, m}(f) \exp \left(-m p \phi^{*}\left(\frac{|\alpha|}{m p}\right)\right) .
$$

Hence $\left(f_{\alpha}\right)_{\alpha \in \mathrm{N}_{0}^{n}}$ is in $\Lambda_{(\omega)}^{\prime}(n)$ and consequently $y:=\left((-i)^{-|\alpha|} f_{\alpha}\right)_{\alpha \in \mathrm{N}_{0}^{n}}$ is also in $\Lambda_{(\omega)}^{\prime}(n)$. Since $T(y)=f$, we proved that $T$ is surjective. Moreover, the estimates above prove also the continuity of $T^{-1}$.

Using Lemma 7(b) and also a slight variation of the arguments above it follows that the assertion of the proposition also holds for $*=\{\omega\}$.

In the sequel we will use the following proposition that follows from Hörmander [6], Theorem 4.4.2.

12. Proposition. For each $n \in \mathrm{N}$ there exist $C_{1}, C_{2}>0$ such that for each plurisubharmonic function $u: \mathrm{C}^{n} \rightarrow \mathrm{R}$ and each $a \in \mathrm{C}^{n}$ there exists $f \in H\left(\mathrm{C}^{n}\right)$ that satisfies

$$
\begin{aligned}
f(a) & =\exp \left(\inf _{|w-a| \leq 1} u(w)-n \log \left(1+|a|^{2}\right)\right) \\
\text { and } \quad|f(z)| & \leq C_{1} \exp \left(\sup _{|w-z| \leq 1} u(w)+C_{2} \log \left(1+|z|^{2}\right)\right), \quad z \in \mathrm{C}^{n} .
\end{aligned}
$$

13. Proposition. Let $\omega$ be a quasianalytic weight function. Then for each $n \in \mathrm{N}$ the space $\mathcal{O}_{0}(n)$ is strictly contained in $\mathscr{E}_{\{\omega\}, 0}(n)$ if and only if $\liminf \operatorname{int}_{t \rightarrow \infty} \frac{\omega(t)}{t}=0$.

Proof. Since $\omega$ satisfies Condition $2(\beta)$, there exists $A \geq 1$ such that $\omega(t) \leq A t+A$ for all $t \geq 0$. If $\liminf _{t \rightarrow \infty} \omega(t) / t>0$ then there exists $B \geq 1$ such that $\omega(t) \geq B t+B$ for all $t \geq 0$. From these two estimates it follows easily that for $y \geq 0$ we have

$$
\phi_{\omega}^{*}(y) \leq B \phi_{t}^{*}\left(\frac{y}{B}\right)-B \quad \text { and } \quad \phi_{t}^{*}(y) \leq A \phi_{\omega}^{*}\left(\frac{y}{A}\right)-A .
$$

Here $\phi_{t}(x)=e^{x}$ denotes the convex function associated to the weight $\sigma(t)=t$. Now (2) implies that $\mathscr{E}_{\{\omega\}}(\Omega)=\mathscr{A}(\Omega)$ for each open set $\Omega \subset \mathrm{R}^{n}$. Hence 
we have $\mathscr{E}_{\{\omega\}, 0}(n)=\mathscr{O}_{0}(n)$ whenever $\liminf _{t \rightarrow \infty} \omega(t) / t>0$. Note that due to analytic continuation, $\mathscr{O}_{0}(n)$ is equal to the space $H(\{0\})$ of all germs of holomorphic functions at the origin. Hence $\mathscr{O}_{0}(n)$ is a (DFN)-space.

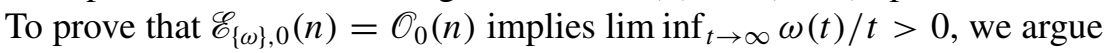
by contradiction and assume that $\mathscr{E}_{\{\omega\}, 0}(n)=\mathscr{O}_{0}(n)$ and $\liminf _{t \rightarrow \infty} \omega(t) / t=$ 0 . Then we choose a sequence $\left(t_{j}\right)_{j} \in \mathrm{N}$ in $] 2, \infty[$ which satisfies

$$
\omega\left(t_{j}\right) \leq \frac{1}{j} t_{j} \quad \text { and } \quad t_{j+1}>2 t_{j}, \quad j \in \mathrm{N} .
$$

Next note that from the second estimate in (2) we get that for each open set $\Omega$ in $\mathrm{R}^{n}$ we have $\mathscr{A}(\Omega) \subset \mathscr{E}_{\{\omega\}}(\Omega)$ with continuous inclusion map. This implies that $\mathscr{O}_{0}(n)$ is continuously embedded in $\mathscr{E}_{\{\omega\}, 0}(n)$. Since it is easy to derive from Corollary 10 that $\mathscr{E}_{\{\omega\}, 0}(n)$ is a (DFS)-space, the open mapping theorem implies that $\mathscr{O}_{0}(n)=\mathscr{E}_{\{\omega\}, 0}(n)$ as locally convex spaces. In particular, they have the same topological dual space. By Corollary 10 this implies that for $\sigma: t \mapsto t$, the spaces $\mathscr{A}_{\{\omega\}, 0}(n)$ and $\mathscr{A}_{\{\sigma\}, 0}(n)$ are equal as Fréchet spaces. Hence the identity map id : $\mathscr{A}_{\{\sigma\}, 0}(n) \rightarrow \mathscr{A}_{\{\omega\}, 0}(n)$ is continuous. Consequently, the following holds:

There exist $p \in \mathrm{N}, p \geq 2$, and $C>0$ such that

$$
\|f\|_{\omega, 1} \leq C\|f\|_{\sigma, p-1}, \quad f \in A_{\{\sigma\}, 0}(n) .
$$

Recall that here we use the notation

$$
\|f\|_{\omega, 1}:=\sup _{z \in C^{n}}|f(z)| \exp (-|\operatorname{Im} z|-\omega(z))
$$

and

$$
\|f\|_{\sigma, p-1}:=\sup _{z \in C^{n}}|f(z)| \exp \left(-\frac{1}{p-1}|z|\right) .
$$

Next we fix $j \in \mathrm{N}$ and we define $\phi_{j}: \mathrm{R} \rightarrow \mathrm{R}$ by

$$
\begin{aligned}
& \left.\left.\phi_{j}(x)=\frac{1}{p} e^{x}, \quad x \in\right]-\infty, \log \left(2 t_{j}\right)\right] \\
& \text { and } \quad \phi_{j}(x)=\frac{2 t_{j}}{p}\left(x-\log \left(2 t_{j}\right)\right)+\frac{2 t_{j}}{p}, \quad x>\log \left(2 t_{j}\right) .
\end{aligned}
$$

Furthermore define $u_{j}: \mathrm{C}^{n} \backslash\{0\} \rightarrow \mathrm{R}, u_{j}(z):=\phi_{j}(\log |z|)$. Since $\phi_{j}$ is convex and continuous, the continuous extension of $u_{j}$ to $C^{n}$ (also denoted by $u_{j}$ ) is plurisubharmonic on $\mathrm{C}^{n}$. Now we apply Proposition 12 to $a_{j}:=\left(t_{j}, 0, \ldots, 0\right)$ and $u=u_{j}$ to get $f_{j} \in H\left(\mathrm{C}^{n}\right)$ satisfying

$$
\begin{aligned}
f_{j}\left(a_{j}\right) & =\exp \left(\inf _{\left|w-a_{j}\right| \leq 1} u_{j}(w)-n \log \left(1+t_{j}^{2}\right)\right) \\
\left|f_{j}(z)\right| & \leq C_{1} \exp \left(\sup _{|w-z| \leq 1} u_{j}(w)+C_{2} \log \left(1+|z|^{2}\right)\right), \quad z \in \mathrm{C}^{n} .
\end{aligned}
$$


Since $u_{j}(t)=t / p$ for $0 \leq t \leq 2 t_{j}$ it follows that for large $j \in \mathrm{N}$ we have

$$
\inf \left\{u_{j}(w):\left|w-a_{j}\right| \leq 1\right\} \geq\left(t_{j}-1\right) / p .
$$

Hence the first estimate in (6) implies the existence of $j_{0} \in \mathrm{N}$ such that for each $j \geq j_{0}$ :

$$
f_{j}\left(a_{j}\right) \geq \exp \left(\frac{t_{j}}{p}\left(1-\frac{1}{t_{j}}-n p \frac{\log \left(1+t_{j}^{2}\right)}{t_{j}}\right)\right) \geq \exp \left(t_{j} / 2 p\right) .
$$

To estimate the second inequality in (6) further, note that by the definition of $u_{j}$ we have for $z \in \mathrm{C}^{n}$ with $|z| \leq 2 t_{j}-1$

$$
\sup \left\{u_{j}(w):|w-z| \leq 1\right\} \leq \frac{|z|+1}{p}=u_{j}(z)+\frac{1}{p} .
$$

For $z \in \mathrm{C}^{n}$ with $|z| \geq 2 t_{j}+1$ we get by the mean-value theorem:

$$
\begin{aligned}
\sup _{|w-z| \leq 1} & \left\{u_{j}(w):|w-z| \leq 1\right\} \\
& \leq \sup _{|w-z| \leq 1}\left\{\frac{2 t_{j}}{p}\left(\log |w|-\log \left(2 t_{j}\right)\right)+\frac{2 t_{j}}{p}\right\} \\
& =u_{j}(z)+\sup \left\{\frac{2 t_{j}}{p}(\log |w|-\log |z|):|w-z| \leq 1\right\} \\
& =u_{j}(z)+\frac{2 t_{j}}{p}(\log (|z|+1)-\log |z|) \leq u_{j}(z)+\frac{2 t_{j}}{p|z|} \\
& \leq u_{j}(z)+\frac{1}{p} .
\end{aligned}
$$

Finally, for $z \in \mathrm{C}^{n}$ with $2 t_{j}-1 \leq|z| \leq 2 t_{j}+1$ and $|w-z| \leq 1$, we get

$$
\begin{aligned}
u_{j}(w) & \leq u_{j}\left(2 t_{j}+1\right)=\phi_{j}\left(\log \left(2 t_{j}+1\right)\right)=\frac{2 t_{j}}{p}\left(\log \left(2 t_{j}+1\right)-\log \left(2 t_{j}\right)\right)+\frac{2 t_{j}}{p} \\
& \leq \frac{1}{p}+\frac{2 t_{j}}{p}=\phi\left(\log \left(2 t_{j}-1\right)\right)+\frac{2}{p}=u_{j}(z)+\frac{2}{p} .
\end{aligned}
$$

Hence we get from (6):

$$
\left|f_{j}(z)\right| \leq C_{1} \exp \left(u_{j}(z)+\frac{2}{p}+C_{2} \log \left(1+|z|^{2}\right)\right), \quad z \in \mathrm{C}^{n} .
$$

From this estimate and the definition of $u_{j}$, it follows that $f_{j}$ is in fact a polynomial. Consequently, $f_{j} \in A_{\sigma, 0}(n)$. Since $u_{j}(t) \leq \frac{1}{p} t$ for all $t \geq 0$ we get from (8) the existence of $C_{3}>0$ such that

$$
\left|f_{j}(z)\right| \leq C_{1} e^{2 / p} \exp \left(|z|\left(\frac{1}{p}+C_{2} \log \left(1+|z|^{2}\right)\right)\right) \leq C_{3} \exp \left(\frac{1}{p-1}|z|\right), \quad z \in \mathrm{C}^{n},
$$

and hence

$$
\left\|f_{j}\right\|_{\sigma, p-1} \leq C_{3} .
$$


Now we get from (4) and (7) that for $j \geq \max \left(j_{0}, 4 p\right)$

$$
C C_{3} \geq\left\|f_{j}\right\|_{1, \omega} \geq f_{j}\left(a_{j}\right) \exp \left(-\omega\left(a_{j}\right)\right) \geq \exp \left(t_{j}\left(\frac{1}{2 p}-\frac{\omega\left(t_{j}\right)}{t_{j}}\right)\right) \geq \exp \left(\frac{t_{j}}{4 p}\right) .
$$

Since $t_{j}$ tends to infinity as $j$ tends to infinity, this inequality is a contradiction. Hence we showed that $\mathscr{O}_{0}(n)=\mathscr{E}_{\{\omega\}, 0}(n)$ implies $\liminf _{t \rightarrow \infty} \frac{\omega(t)}{t}>0$.

14. Theorem. Let $\omega$ be a quasianalytic weight function. Then the Borel map

$$
B: \mathscr{E}_{\{\omega\}, 0}(n) \rightarrow \Lambda_{\{\omega\}}(n), B(f):=\left(f^{(\alpha)}(0)\right)_{\alpha \in \mathrm{N}_{0}^{n}}
$$

is surjective if and only if $\liminf _{t \rightarrow \infty} \frac{\omega(t)}{t}>0$.

Proof. Since $B$ is linear and continuous, its adjoint $B^{t}: \Lambda_{\{\omega\}}^{\prime}(n) \rightarrow$ $\mathscr{E}_{\{\omega\}, 0}^{\prime}(n)$ is continuous, too. Now note that by Corollary 10 the Fourier-Laplace transform $\mathscr{F}: \mathscr{E}_{\{\omega\}, 0}^{\prime}(n) \rightarrow A_{\{\omega\}, 0}(n)$ is a linear topological isomorphism, and that by Proposition 11 the map $T: \Lambda_{\{\omega\}}^{\prime}(n) \rightarrow A_{\{\omega\}}(n)$ is a linear topological isomorphism. Hence the map $\mathscr{F} \circ B^{t}: \Lambda_{\{\omega\}}^{\prime}(n) \rightarrow A_{\{\omega\}, 0}(n)$ also has this property. To compute it, fix $y=\left(y_{\alpha}\right)_{\alpha \in \mathrm{N}_{0}^{n}}$ in $\Lambda_{\{\omega\}}^{\prime}(n)$. Then we get

$$
\begin{aligned}
\mathscr{F} \circ B^{t}(y) & =\left\langle B^{t}(y)_{x}, \exp (-i\langle x, y,\rangle)\right\rangle=\left\langle y, B_{x}(\exp (-i\langle x, z\rangle))\right\rangle \\
& =\sum_{\alpha \in \mathrm{N}_{0}^{n}} y_{\alpha}(-i)^{|\alpha|} z^{\alpha}=T(y) .
\end{aligned}
$$

This shows that $\mathscr{F} \circ B^{t}=J \circ T$, where $J$ denotes the obvious inclusion map $A_{\{\omega\}}(n) \hookrightarrow A_{\{\omega\}, 0}(n)$.

If we assume now that $\lim _{i n f} \operatorname{in}_{t \rightarrow \infty} \omega(t) / t>0$ then this and Condition $2(\beta)$ imply the existence of $A \in \mathrm{N}$ such that

$$
t \leq A \omega(t)+A \quad \text { and } \quad \omega(t) \leq A t+A, \quad t \geq 0 .
$$

From these two estimates we get for each $k \in \mathrm{N}$ :

(9) $\frac{1}{k} \omega(z) \leq \frac{1}{k}(|\operatorname{Im} z|+\omega(z)) \leq \frac{1}{k}(1+A)|z|+\frac{A}{k} \leq \frac{1+A}{k} A \omega(z)+\frac{A(1+A)+A}{k}$.

It is easy to check that these estimates imply that

$$
A_{\{\omega\}, 0}(n)=A_{\{\omega\}}(n)
$$

as Fréchet spaces. Hence $B^{t}=\mathscr{F}^{-1} \circ J \circ T$ is a linear topological isomorphism. Since the spaces $\Lambda_{\{\omega\}}(n)$ and $\mathscr{E}_{\{\omega\}, 0}(n)$ are reflexive, it follows that $B$ is an isomorphism and hence surjective, whenever $\liminf _{t \rightarrow \infty} \omega(t) / t>0$.

To show that $B$ is not surjective for each quasianalytic weight function that satisfies $\liminf _{t \rightarrow \infty} \omega(t) / t=0$, we argue by contradiction, i.e., we assume 
that the latter condition holds and that $B$ is surjective. Then the considerations above show that $J=\mathscr{F} \circ B^{t} \circ T^{-1}$ is a linear topological isomorphism. Hence $\mathscr{A}_{\{\omega\}}(n)$ and $A_{\{\omega\}, 0}(n)$ are equal as Fréchet spaces.

Next we define for $m \in \mathrm{N}$ the Banach spaces

$$
E_{m}:=\left\{f \in H\left(C^{n}\right):\|f\|_{m}:=\sup _{z \in C^{n}}|f(z)| \exp \left(-\frac{1}{m}(|\operatorname{Im} z|+\omega(z))\right)<\infty\right\}
$$

and

$$
E_{m}^{0}=\left\{f \in H\left(C^{n}\right): \lim _{|z| \rightarrow \infty} f(z) \exp \left(-\frac{1}{m}(|\operatorname{Im} z|+\omega(z))\right)=0\right\} \subset E_{m} .
$$

Then $A_{\{\omega\}, 0}(n)=\operatorname{proj}_{\leftarrow m} E_{m}^{0}$ and the projective spectrum is reduced, i.e., $A_{\{\omega\}, 0}(n)$ is dense in $E_{m}^{0}$ for each $m \in \mathrm{N}$. To get this, we note first that for each $m \in \mathrm{N}$ the function $\phi(z):=\frac{1}{m}(|\operatorname{Im} z|+\omega(z))$ satisfies the conditions (i)-(v) in Taylor [13], Section 3. Since $\omega$ is quasianalytic, $\phi$ also satisfies Condition (3.4) in [13]. Hence [13], Theorem 6, implies that the polynomials are dense in $E_{m}^{0}$. Of course, this shows that $A_{\{\omega\}, 0}(n)$ is dense in $E_{m}^{0}$. From this fact and the identity $A_{\{\omega\}}(n)=A_{\{\omega\}, 0}(n)$, we get in particular the existence of $m \in \mathrm{N}$ and $C>0$ such that

$$
\begin{aligned}
\sup _{z \in C^{n}}|f(z)| e^{-\omega(z)} & =\|f\|_{\{0\}, 1} \leq C\|f\|_{m} \\
& =\sup _{z \in C^{n}}|f(z)| \exp \left(-\frac{1}{m}(|\operatorname{Im} z|+\omega(z))\right)
\end{aligned}
$$

holds for all $f \in A_{\{\omega\}, 0}(n)$ and even for all $f \in E_{m}^{0}$. See Definition 8(b) and 8(c) for the definition of $\|\cdot\|_{\{0\}, 1}$.

To show that the estimate in (10) does not hold for all $f \in E_{m}^{0}$, we choose $K \in \mathrm{N}$ such that Condition $2(\alpha)$ holds for $K$. This implies, for each $a, b \geq 0$, that $\omega(a+b) \leq K \omega(a)+K \omega(b)+K$. Since we assume that $\liminf \operatorname{in}_{t \rightarrow \infty} \omega(t) / t=0$, we can find a sequence $\left(t_{j}\right)_{j \in \mathrm{N}}$ in $[1, \infty[$ such that

$$
\omega\left(t_{j}\right) \leq \frac{1}{j} t_{j} \quad \text { and } \quad t_{j+1}>2 t_{j}, \quad j \in \mathrm{N} .
$$

Next we apply Proposition 12 with $a_{j}:=\left(i t_{j}, 0, \ldots, 0\right)$ and $u: z \mapsto$ $\frac{1}{p}(|\operatorname{Im} z|+\omega(z))$, where $p:=(K+1)(m+1)$ to get $f_{j} \in H\left(C^{n}\right)$ which satisfies

$$
\begin{aligned}
f_{j}\left(a_{j}\right) & =\exp \left(\inf _{\left|w-a_{j}\right| \leq 1} u_{j}(w)-n \log \left(1+\left|a_{j}\right|^{2}\right)\right), \\
\left|f_{j}(z)\right| & \leq C_{1} \exp \left(\sup _{|w-z| \leq 1} u_{j}(w)+C_{2} \log \left(1+|z|^{2}\right)\right) .
\end{aligned}
$$


Now note that

$$
\begin{aligned}
\inf _{\left|w-a_{j}\right| \leq 1}(|\operatorname{Im} w|+\omega(w)) & \geq\left|\operatorname{Im} a_{j}\right|-1+\frac{1}{K} \omega\left(a_{j}\right)-\omega(1)-1 \\
& =t_{j}+\frac{1}{K} \omega\left(t_{j}\right)-(2+\omega(1))
\end{aligned}
$$

and

$$
\sup _{|w-z| \leq 1}|\operatorname{Im} w|+\omega(w) \leq|\operatorname{Im} z|+1+K \omega(z)+K \omega(1)+K
$$

From these estimates and (12) we now get the existence of $j_{0} \in \mathbf{N}$ such that for $j \geq j_{0}$ we have

$$
\begin{aligned}
f_{j}\left(a_{j}\right) & \geq \exp \left(\frac{1}{p} t_{j}-(2+\omega(1))-n \log \left(1+t_{j}^{2}\right)\right) \geq \exp \left(\frac{t_{j}}{2 p}\right) \\
& =\exp \left(\frac{t_{j}}{2(K+1)(m+1)}\right) . \\
\left|f_{j}(z)\right| & \leq C_{1} e^{(1+K+\omega(1)) / p} \exp \left(\frac{1}{p}|\operatorname{Im} z|+\frac{K}{p} \omega(z)+C_{2} \log \left(1+|z|^{2}\right)\right) \\
& \leq C_{3} \exp \left(\frac{K+1}{p}(|\operatorname{Im} z|+\omega(z))\right)=C_{3} \exp \left(\frac{1}{m+1}(|\operatorname{Im} z|+\omega(z))\right),
\end{aligned}
$$

where we used Condition $2(\gamma)$ to get the last estimate. The last estimate implies that $f_{j}$ is in $E_{m}^{0}$ and that $\left\|f_{j}\right\|_{m} \leq C_{3}$ for all $j$. From (13) and (10) we now get for $j \geq j_{0}$

$$
\begin{aligned}
C C_{3} & \geq C\left\|f_{j}\right\|_{m} \geq\left\|f_{j}\right\|_{\{0\}, 1} \geq\left|f_{j}\left(a_{j}\right)\right| \exp \left(-\omega\left(a_{j}\right)\right) \\
& \geq \exp \left(t_{j}\left(\frac{1}{2(K+1)(m+1)}-\frac{\omega\left(t_{j}\right)}{t_{j}}\right)\right)
\end{aligned}
$$

However, this estimate does not hold for large $j$, since by (11), the sequence on the right hand side above tends to infinity. Hence $B$ is not surjective if the quasianalytic weight function satisfies $\liminf _{t \rightarrow \infty} \omega(t) / t=0$.

Combining Proposition 13 and Theorem 14 we get the following.

15. Corollary. Let $\omega$ be a quasianalytic weight function. Then the Borel map

$$
B: \mathscr{E}_{\{\omega\}, 0}(n) \rightarrow \Lambda_{\{\omega\}}(n), \quad B(f):=\left(f^{\alpha}(a)\right)_{\alpha \in \mathrm{N}_{0}^{n}}
$$

is surjective if and only if $\mathscr{E}_{\{\omega\}, 0}(n)=\mathscr{O}_{0}(n)$.

REMARK. Corollary 15 shows that a classical result of Carleman for the quasianalytic classes $\mathscr{E}_{\left\{M_{p}\right\}}$ also holds if one defines the classes using weight functions $\omega$. For the statement and a modified proof of Carleman's result we refer to Thillez [14], Theorem 3. 
16. Corollary. Let $\omega$ be a quasianalytic weight function. Then for each open set $\Omega$ in $\mathrm{R}^{n}$ which contains the origin, the Borel map

$$
\left.B: \mathscr{E}_{\{\omega\}}(\Omega) \rightarrow \Lambda_{\{\omega\}}(n), \quad B(f):=\left(f^{(\alpha}\right)(0)\right)_{\alpha \in \mathrm{N}_{0}^{n}}
$$

is not surjective.

Proof. If $\omega$ satisfies $\liminf _{t \rightarrow \infty} \omega(t) / t=0$, this follows immediately from Theorem 14, since $\mathscr{E}_{\{\omega\}}(\Omega)$ is a subspace of $\mathscr{E}_{\{\omega\}, 0}(n)$.

If $\lim \inf _{t \rightarrow \infty} \omega(t) / t>0$ then we showed at the beginning of the proof of Proposition 13 that $\mathscr{E}_{\{\omega\}}(\Omega)=\mathscr{A}(\Omega)$ for each open set $\Omega$ in $\mathrm{R}^{n}$. If $0 \in \Omega$ then we choose $r>0$ such that $\overline{B(0, r)} \subset \Omega$ and we define

$$
g: B(0, r) \rightarrow \mathrm{R}, \quad g(x):=\exp \left(\frac{-1}{r^{2}-|x|^{2}}\right) .
$$

We also define $b \in \Lambda_{\{\omega\}}(n)$ by $b_{\alpha}:=g^{(\alpha)}(0), \alpha \in \mathrm{N}_{0}^{n}$, and we claim that $b$ is not in the range of $B$. To see this, assume that there is $f \in \mathscr{E}_{\{\omega\}}(\Omega)=\mathscr{A}(\Omega)$ with $B(f)=b$. Then the uniqueness theorem for real-analytic functions implies that $f=g$ on the connected component of $\Omega$ which contains $B(0, r)$. Hence $f$ is a real-analytic extension of $g$ to a neighborhood of $\overline{B(0, r)}$. Since no such extension exists, the map $B: \mathscr{E}_{\{\omega\}}(\Omega) \rightarrow \Lambda_{\{\omega\}}(n)$ is not surjective.

17. Remark. Corollary 16 shows in particular that the Borel map

$$
B: \mathscr{A}\left(\mathrm{R}^{n}\right)=\mathscr{E}_{\{p !\}}\left(\mathrm{R}^{n}\right) \rightarrow \Lambda_{\{p !\}}(n)=\Lambda_{\{t\}}(n)
$$

is not surjective. This shows that the statement of Chung and Kim [5], Theorem 3.2 , is not correct.

18. Proposition. Let $\omega$ be a quasianalytic weight function. Then for each $n \in \mathrm{N}$ the space $H\left(\mathrm{C}^{n}\right)$ is strictly contained in $\mathscr{E}_{(\omega), 0}(n)$ if and only if $\liminf _{t \rightarrow \infty} \omega(t) / t=0$.

Proof. If $\lim \inf _{t \rightarrow \infty} \omega(t) / t>0$ then we showed in the proof of Proposition 13 that (2) holds. This implies that for $\sigma: t \mapsto t$ we have $\mathscr{E}_{(\omega), 0}(n)=$ $\mathscr{E}_{(\sigma), 0}(n)$. Since $\mathscr{E}_{(\sigma)}(\Omega)=H\left(C^{n}\right)$ for each open set $\Omega$, it follows that $\mathscr{E}_{(\omega), 0}(n)=H\left(C^{n}\right)$.

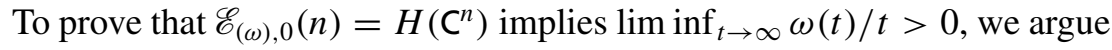
by contradiction and assume that $\lim _{i n f} \ln _{t \rightarrow \infty} \omega(t) / t=0$. Then we choose a sequence $\left(t_{j}\right)_{j \in \mathrm{N}}$ in ]1, $\infty$ [ which satisfies (3). Since $H\left(\mathrm{C}^{n}\right)$ is continuously embedded in $\mathscr{E}_{(\omega)}(\Omega)$ and hence in $\mathscr{E}_{(\omega), 0}(n)$, the open mapping theorem implies that the topologies of $H\left(C^{n}\right)=\mathscr{E}_{(\sigma), 0}(n)$ and $\mathscr{E}_{(\omega), 0}(n)$ coincide. We apply Corollary 10 to conclude that the spaces $A_{(\sigma), 0}(n)=A_{(\sigma)}(n)$ and $A_{(\omega), 0}(n)$ are equal as locally convex spaces. Hence the identity map id : $A_{(\sigma)}(n) \rightarrow$ 
$A_{(\omega), 0}(n)$ is continuous. This implies that for each bounded set $B$ in $A_{(\sigma)}(n)$, $B$ is bounded in $A_{(\omega), 0}(n)$. In particular, for each $m \in \mathrm{N}$ there exist $k \in \mathrm{N}$ and $C>0$ such that for each $f \in A_{(\sigma)}(n)$ which satisfies $|f(z)| \leq \exp (2|z|)$, $z \in \mathrm{C}^{n}$, also satisfies

$$
|f(z)| \leq C \exp \left(\frac{1}{m}|\operatorname{Im} z|+k \omega(z)\right), \quad z \in \mathbb{C}^{n} .
$$

We fix $m=1$ and choose $k$ and $C$ accordingly. Then we define $\phi_{j}: \mathrm{R} \rightarrow \mathrm{R}$ by

$$
\begin{aligned}
& \left.\left.\phi_{j}(x):=e^{x}, \quad x \in\right]-\infty, \log \left(2 t_{j}\right)\right] \\
& \text { and } \quad \phi_{j}(x):=2 t_{j}\left(x-\log \left(2 t_{j}\right)\right)+2 t_{j}, \quad x>\log \left(2 t_{j}\right) \text {. }
\end{aligned}
$$

Furthermore we define $u_{j}: C^{n} \backslash\{0\} \rightarrow R, u_{j}(z):=\phi_{j}(\log |z|)$. Since $\phi_{j}$ is convex and continuous, the continuous extension of $u_{j}$ to $C^{n}$ (also denoted by $u_{j}$ ) is plurisubharmonic on $C^{n}$. Now we apply Proposition 12 to $a_{j}:=$ $\left(t_{j}, 0, \ldots, 0\right)$ and $u=u_{j}$ to get $f_{j} \in H\left(\mathrm{C}^{n}\right)$ satisfying

$$
\begin{aligned}
f_{j}\left(a_{j}\right) & =\exp \left(\inf _{\left|w-a_{j}\right| \leq 1} u(w)-n \log \left(1+t_{j}^{2}\right)\right) \\
\left|f_{j}(z)\right| & =C_{1} \exp \left(\sup _{|w-z| \leq 1} u(w)+C_{3} \log \left(1+|z|^{2}\right)\right), \quad z \in \mathrm{C}^{n} .
\end{aligned}
$$

As in the proof of Proposition 13 it follows from these estimates that there exists $j_{0} \in \mathrm{N}$ that for each $j \geq j_{0}$ we have

$$
\begin{aligned}
f_{j}\left(a_{j}\right) & \geq \exp \left(t_{j}\left(1-\frac{1}{t_{j}}\left(1+n \log \left(1+t_{j}\right)\right)\right)\right) \geq \exp \left(t_{j} / 2\right), \\
\left|f_{j}(z)\right| & \leq C_{1} \exp \left(u_{j}(z)+2+C_{2} \log \left(1+|z|^{2}\right)\right), \quad z \in \mathrm{C}^{n} .
\end{aligned}
$$

Since $\phi_{j}(x) \leq \phi_{\sigma}(x)$ for all $x \in \mathrm{R}$, it follows from (17) that there exists $C_{3}>0$ such that

$$
\left|f_{j}(z)\right| \leq C_{3} \exp (2|z|), \quad z \in \mathrm{C}^{n} .
$$

Now our assumption implies that from (14) and (17) we get for each $j \geq j_{0}$

$$
\begin{aligned}
C_{3} C & \geq \sup _{z \in C^{n}}\left|f_{j}(z)\right| \exp (-|\operatorname{Im} z|-k \omega(z)) \geq f_{j}\left(a_{j}\right) \exp \left(-k \omega\left(t_{j}\right)\right) \\
& \geq \exp \left(t_{j}\left(\frac{1}{2}-\frac{k \omega\left(t_{j}\right)}{t_{j}}\right)\right) .
\end{aligned}
$$

Since we assumed that $\lim _{j \rightarrow \infty} \omega\left(t_{j}\right) / t_{j}=0$ and since the sequence $\left(t_{j}\right)_{j \in \mathrm{N}}$ tends to infinity, this estimate cannot hold for large $j \in \mathrm{N}$. This contradiction completes the proof of the proposition.

19. Theorem. Let $\omega$ be a quasianalytic weight function. Then the Borel map

$$
B: \mathscr{E}_{(\omega), 0}(n) \rightarrow \Lambda_{(\omega)}(n), \quad B(f):=\left(f^{(\alpha)}(0)\right)_{\alpha \in \mathrm{N}_{0}^{n}}
$$

is surjective if and only if $\lim _{\inf } \rightarrow \infty \omega(t) / t>0$. 
Proof. Since $B$ is linear and continuous, its adjoint $B^{t}: \Lambda_{(\omega)}^{\prime}(n) \rightarrow$ $\mathscr{E}_{(\omega), 0}^{\prime}(n)$ is continuous, too. By Corollary 10 the Fourier-Laplace transform $\mathscr{F}: \mathscr{E}_{(\omega), 0}^{\prime}(n) \rightarrow A_{(\omega), 0}^{\prime}(n)$ is a linear topological isomorphism and by Proposition 11 the map $T: \Lambda_{(\omega)}^{\prime}(n) \rightarrow A_{(\omega)}(n)$ has the same property. Consequently, $\mathscr{F} \circ B^{t}: \Lambda_{(\omega)}^{\prime}(n) \rightarrow A_{(\omega), 0}(n)$ is a linear topological isomorphism. As in the proof of Theorem 14. we get that $\mathscr{F} \circ B^{t}=J \circ T$, where $J: A_{(\omega)}(n) \hookrightarrow A_{(\omega), 0}(n)$ denotes the inclusion map.

If we assume that $\liminf _{t \rightarrow \infty} \omega(t) / t>0$ then it follows again that the estimates (9) hold. It is easy to check that they imply

$$
A_{(\omega), 0}(n)=A_{(\omega)}(n) .
$$

Hence $B^{t}=\mathscr{F}^{-1} \circ J \circ T$ is a linear topological isomorphism. Since the spaces $\Lambda_{(\omega)}(n)$ and $\mathscr{E}_{(\omega), 0}(n)$ are reflexive, $B$ is an isomorphism whenever $\lim \inf _{t \rightarrow \infty} \omega(t) / t>0$.

To show that $B$ is not surjective for each quasianalytic weight function that satisfies $\liminf _{t \rightarrow \infty} \omega(t) / t=0$, we argue by contradiction, i.e., we assume that the latter condition holds and that $B$ is surjective. Since $\omega$ is quasianalytic, $B$ is also injective. Hence the open mapping theorem implies that $B$ is a linear topological isomorphism. Now we apply Gothendieck's factorization theorem (see Meise and Vogt [10], 24.33) to get $q \in \mathrm{N}$ such that $B^{-1}\left(\Lambda_{(\omega)}(n)\right) \subset$ $\mathscr{E}_{(\omega)}\left(B\left(0, \frac{1}{q}\right)\right)$. Since the quasianalyticity of $\omega$ implies that for $k>q$ the restriction $\rho_{q, k}: \mathscr{E}_{(\omega)}\left(B\left(0, \frac{1}{q}\right)\right) \rightarrow \mathscr{E}_{(\omega)}\left(B\left(0, \frac{1}{k}\right)\right)$ is injective, we get that $\mathscr{E}_{(\omega), 0}(n)=\mathscr{E}_{(\omega)}\left(B\left(0, \frac{1}{q}\right)\right)$. Moreover, if we define for $m \in \mathrm{N}$

$$
\mathscr{E}_{(\omega), m}(n):=\left\{f \in C^{\infty}(\overline{B(0,1 / m)}): \text { For each } k \in \mathbf{N}: p_{k, m}(f)<\infty\right\}
$$

and

$$
\begin{aligned}
\mathscr{A}_{(\omega), m}(n):=\left\{f \in H\left(C^{n}\right): \text { There is } k \in \mathrm{N}:\right. \\
\left.\|f\|_{k}:=\sup _{z \in C^{n}}|f(z)| \exp \left(-\frac{1}{m}|\operatorname{Im} z|-k \omega(z)\right)<\infty\right\}
\end{aligned}
$$

then we can choose $m \in \mathrm{N}, m>q$, such that $\mathscr{E}_{(\omega), 0}(n)=\mathscr{E}_{(\omega), m}(n)$. Then

$$
B_{m}: \mathscr{E}_{(\omega), m}(n) \rightarrow \Lambda_{(\omega)}(n), \quad B_{m}=\left.B\right|_{\mathscr{E}_{(\omega), m}(n)}
$$

is a linear topological isomorphism. Next note that the proof of Meise and Taylor [9], Proposition 3.6, shows also in the present case that the FourierLaplace transform

$$
\mathscr{F}: \mathscr{E}_{(\omega), m}^{\prime}(n) \rightarrow \mathscr{A}_{(\omega), m}(n), \quad \mathscr{F}(\mu)[z]:=\left\langle\mu_{x}, \exp (-i\langle x, z\rangle)\right\rangle,
$$


is surjective. From the above it follows that $\mathscr{F}$ is in fact an isomorphism. As in the first part of the proof we now get that $\mathscr{F} \circ B_{m}^{t}=J_{m} \circ T$, where $J_{m}$ denotes the inclusion of $A_{(\omega)}(n)$ in $A_{(\omega), m}(n)$. Hence $J_{m}$ is a linear topological isomorphism. Since $A_{(\omega)}(n)$ and $A_{(\omega), m}(n)$ are (DFN)-spaces, it follows that each set $B$ which is bounded in $A_{(\omega), m}(n)$ is bounded in $A_{(\omega)}(n)$. Now choose $K \in \mathrm{N}$ such that Condition $2(\alpha)$ holds for $\omega$. Then define

$$
B:=\left\{f \in A_{(\omega), m}(n):\|f\|_{K+1} \leq 1\right\} .
$$

Since $B$ is bounded in $A_{(\omega), m}(n)$, there must exist $l \in \mathrm{N}$ such that

$$
\sup _{f \in B} \sup _{z \in C^{n}}|f(z)| \exp (-l \omega(z))<\infty .
$$

Since we assumed that $\liminf _{t \rightarrow \infty} \omega(t) / t=0$, we can choose a sequence $\left(t_{j}\right)_{j \in \mathrm{N}}$ in $\left[1, \infty\right.$ [ that satisfies (11). Then we apply Proposition 12 with $a_{j}=$ $\left(i t_{j}, 0, \ldots, 0\right)$ and $u(z):=\frac{1}{m}|\operatorname{Im} z|+\omega(z)$ to get $f_{j} \in H\left(C^{n}\right)$ satisfying

$$
\begin{aligned}
f_{j}\left(a_{j}\right) & \geq \exp \left(\inf _{\left|w-a_{j}\right| \leq 1} u(w)-n \log \left(1+t_{j}^{2}\right)\right) \\
\left|f_{j}(z)\right| & \leq C_{1} \exp \left(\sup _{|w-z| \leq 1} u(w)+C_{2} \log \left(1+|z|^{2}\right)\right) .
\end{aligned}
$$

As in the proof of Theorem 14 these estimates imply the existence of $j_{0} \in \mathbf{N}$ such that for $j \geq j_{0}$ we have

$$
\begin{aligned}
f_{j}\left(a_{j}\right) \geq & \exp \left(\frac{1}{m} t_{j}-\frac{2+\omega(1)}{m}-\frac{1}{K} \omega\left(t_{j}\right)\right) \geq \exp \left(t_{j} / 2 m\right) \\
\left|f_{j}(z)\right| \leq & C_{1} \exp \left(\frac{1}{m}|\operatorname{Im} z|+\frac{1}{m}+K \omega(z)\right. \\
& \left.\quad+K \omega(1)+K+C_{2} \log \left(1+|z|^{2}\right)\right) \\
& \quad C_{3} \exp \left(\frac{1}{m}|\operatorname{Im} z|+(K+1) \omega(z)\right), \quad z \in \mathrm{C}^{n} .
\end{aligned}
$$

From (20) we see that the sequence $\left(f_{j}\right)_{j \in \mathrm{N}}$ is in $C_{3} B$. Hence we can apply (18), which gives

$$
\begin{aligned}
\infty & >\sup _{j \in \mathbb{N}} \sup _{z \in C^{n}}\left|f_{j}(z)\right| \exp (-l \omega(z)) \\
& \geq \sup _{j \geq j_{0}}\left|f_{j}\left(a_{j}\right)\right| \exp \left(-l \omega\left(a_{j}\right)\right) \\
& \geq \sup _{j \geq j_{0}} \exp \left(t_{j}\left(\frac{1}{2 m}-\frac{l \omega\left(t_{j}\right)}{t_{j}}\right)\right) .
\end{aligned}
$$

Since the right hand side of this estimate tends to infinity as $j$ tends to infinity, we derived a contradiction. 
20. Corollary. Let $\omega$ be a quasianalytic weight function. Then the Borel map

$$
B: \mathscr{E}_{(\omega), 0}(n) \rightarrow \Lambda_{(\omega)}(n)
$$

is surjective if and only if $\mathscr{E}_{(\omega), 0}(n)=H\left(\mathrm{C}^{n}\right)$.

ACKNOWLEDGEMENT. The research of Bonet was partially supported by MEC and FEDER Project MTM2010-15200 and by GV Project Prometeo /2008/101.

\section{REFERENCES}

1. Bonet, J., Meise, R., Taylor, B. A., Whitney's extension theorem for ultradifferentiable functions of Roumieu type, Proc. Roy. Irish Acad. (A) 89 (1989), 53-66.

2. Bonet, J., Meise, R., Taylor, B. A., On the range of the Borel map for classes of nonquasianalytic functions, pp. 97-111 in: Progress in Functional Analysis, Proc. Peñíscola 1990, North Holland Math. Studies 170, North Holland, Amsterdam 1992.

3. Bonet, J., Meise, R., Melikhov, S. N., A comparison of two different ways to define classes of ultradifferentiable functions, Bull. Belg. Math. Soc. Simon Stevin 14 (2007), 425-444.

4. Braun, R., Meise, R., Taylor, B. A., Ultradifferentiable functions and Fourier analysis, Results Math. 17 (1990), 206-237.

5. Chung, S. Y., Kim, D., Between hyperfunctions and ultradistributions, Bull. Cl. Sci. Math. Nat. Sci. Math. 24 (1999), 89-106.

6. Hörmander, L., An Introduction to Complex Analysis in Several Variables, North Holland Math. Library 7, North Holland, Amsterdam 1990.

7. Heinrich, T., Meise, R.: A support theorem for quasianalytic functionals, Math. Nachr. 280 (2007), 364-387.

8. Komatsu, H., Ultradistributions I, structure theorems and a characterization, J. Fac. Sci. Tokyo (IA) 20 (1973), 25-105.

9. Meise, R., Taylor, B. A., Whitney's extension theorem for ultradifferentiable functions of Beurling type, Ark. Mat. 26 (1988), 265-287.

10. Meise, R., Vogt, D., Introduction to Functional Analysis, Oxford Grad. Texts in Math. 2, Clarendon Press, Oxford 1997.

11. Petzsche, H., On E. Borel's theorem, Math. Ann. 282 (1988), 299-313.

12. Rösner, Th., Surjektivität partieller Differentialoperatoren auf quasianalytischen RoumieuKlassen, Diss., Heinrich-Heine-Univ. Düsseldorf 1997.

13. Taylor, B. A., On weighted polynomial approximation of entire functions, Pacific J. Math. 36 (1971), 523-539.

14. Thilliez, V., On quasianalytic local rings, Expo. Math. 26 (2008), 1-23.

INSTITUTO UNIVERSITARIO

DE MATEMÁTICA PURA Y APLICADA

UNIVERSITAT POLITĖCNICA DE VALÈNCIA

E-46071 VALENCIA

SPAIN

E-mail: jbonet@mat.upv.es
MATHEMATISCHES INSTITUT

HEINRICH-HEINE-UNIVERSITÄT

UNIVERSITÄTSSTRAßE 1

D-40225 DÜSSELDORF

GERMANY

E-mail: meise@math.uni-duesseldorf.de 